\title{
Research on Animation Majors' Competition and Cultivation of Creative Talents
}

\author{
Ping Guan ${ }^{1, *}$ Zeling Zheng ${ }^{1}$ \\ ${ }^{1}$ School of Art and Design, Fuzhou College of Foreign Studies and Trade, Fuzhou, Fujian 350018, China \\ *Corresponding author.Email: 37754129@qq.com
}

\begin{abstract}
With the booming development of the animation industry, the demand for creative talents in the animation industry is also increasing. As a high-level platform for the training of creative talents in the animation industry, universities have undoubtedly affected the prospects and future of the animation industry. This article starts from the perspective of analyzing the characteristics of the animation industry and the current status of animation teaching in colleges and universities, and discusses the role of college competitions in the teaching reform and creative talent training of animation majors. Meanwhile, the authors of this article put forward a set of practical teaching mode with competition as the engine, combine school-enterprise cooperation, work-study combination, and carry out teaching reforms to improve teaching quality and hope to meet the purpose of training high-quality animation creative talents.
\end{abstract}

\section{Keywords: animation and cartoon, competitions, creative talent training}

\section{INTRODUCTION}

The animation industry is one of the most vigorous emerging cultural industries since the twentieth century. It is a high-quality medium that re-innovates the country's traditional culture and national folk culture, and is an important choice for the effective development and transformation of the regional economy. In recent years, governments at all levels have issued corresponding policies to actively support and promote the development of the animation industry. For example, in the Fujian Province on the west coast of the Straits, its animation industry has begun to flourish, and a large number of outstanding animation and entrepreneurial industrial parks have emerged, such as Rongdu 318 Cultural Creative Art Street, Fuzhou "Taiwan Creative Park", "Haixi Animation Creative Park", etc. The output of Fuzhou's original animation and games has entered the top ten in China, and the cultural and creative industries have become the supporting industries for regional economic development. In 2018, the cultural and creative industries in Fujian province achieved an added value of 60 billion yuan, accounting for $4.5 \%$ of local GDP reaching a new high in history. Fujian Province's animation industry has entered its best period, with

*Project: Research Project on Educational Reform of Undergraduate Education in Fujian Province "Research on Innovation of Animation Major from the Perspective of Fujian Cultural Industry Cluster" (Project No.: FBJG20190283).

CLC number: G424

Document number: A output increasing exponentially, with a wide variety of animation products, which meets multi-level needs. However, there are still few outstanding works, and there is still a large gap in overall quality, character, and creativity; great efforts are still required. Its core and bottlenecks are all in the "talent", and in the future it will force a greater number of talent needs, especially the comprehensive, high-quality professional talents, creative talents, and technical talents in the animation industry.

In modern society, talents are mainly cultivated by scientific research institutes in universities. The rapid development of the animation industry is always inseparable from talents, especially high-quality, innovative and creative talents. Colleges and universities are treated as important training bases; the quality of professional talents is directly related to the prospects and future of the industry. Although many universities have made many attempts to reform and put forward many teaching models, they are still far from the actual needs, mainly reflected in the animation talents cultivated by the universities. No matter in terms of practical skills, innovative ideas, and teamwork spirit, it is still in a state of inadequate professionalism when entering the society. Some parts are difficult to meet the corresponding job requirements, and it is difficult to truly meet the increasingly fierce market demands of social competition. Based on this basic situation, colleges and universities should absorb various lessons and actively explore system reforms and innovations in the practice teaching mode of 
animation. Efforts should be made to integrate competitive teaching with practical internship methods and classroom teaching methods, strengthen students' ability to expand design thinking, teamwork, professional software tools and practical skills, and deepen the reform of the teaching curriculum and system, the reform of the evaluation system of teaching achievements, and the animation creative high-quality talent training model that meets the needs of the new era in many ways. [1]

\section{STATUS OF PRACTICAL TEACHING IN ANIMATION}

The focus of animation teaching is in colleges and universities. The focus of professional teaching in colleges and universities includes the basic concepts, theories, and tools of animation, as well as the techniques and practices of animation design and production. They should also make students have a comprehensive grasp of the basic theory of animation design and production, and be proficient in using contemporary mainstream animation production tools, such as FLASH, 3DMAX, etc. The students can learn some basic modeling tools, have certain creative ability, expression ability, organization ability, especially in script editing, plot design, scene planning, character design, material production, short video editing and animation special effects in film and television animation, to make them have a good professional level and reach the application and comprehensive animation creative level. In addition, on the basis of conveying professional theoretical knowledge, colleges and universities put more emphasis on applying what they have learned, using internships instead of classroom exercises, attaching more importance to training practical skills and animation script planning skills, and adding more professional animation plot design capabilities, character and scene modeling capabilities, software tool drawing capabilities, and story creative imagination. However, due to various reasons, the current teaching methods are difficult to achieve the expected results. To sum up, there are several problems, which greatly affect the expected results of the animation professional training [2].

\section{A. Practice content is simple and modular}

In the actual work created by the corporate team, an excellent animation work requires a well-organized and orderly team and close cooperation between members to create it. However, at present, the practice teaching of animation majors in colleges and universities generally has a simple content problem. Teachers often only arrange independent and single homework or extracurricular exercises, which is only enough for a single person. Even if there are more complicated assignments that require three or five team members to complete, in fact due to the lack of monitoring and team self-discipline, they often rely on one or two people in the team to do it. The other members have basically nothing to do and it is difficult to reach expected results of teamwork exercises.

Compared with out-of-school practical training, the content of in-school practical teaching often comes from the teachers 'own ideas, their own design, or their creativity, which is relatively simple. Moreover, the teaching content in colleges and universities is relatively fixed, forming a modelized and rigid teaching thinking mode. As a result, students have relatively basic design methods and practical skills, imitate and innovate creatively, complete utilitarian tasks and pass exams, and lack originality and high-end creativity, resulting in a lack of exercise of creative and creative thinking and slow improvement.

\section{B. Internship and practical training are inconsistent}

The animation major is different from the general disciplines. It is very practical. Many professional courses have a large proportion of practical time, and there are many practical links for students to participate in. However, in the specific teaching process, these practical links lack connection, are relatively independent, and are not effectively integrated. They often complete tasks in stages. Therefore, when students use tool software to design and produce, they are often too busy with current tasks and ignore overall and systematic design exercises. They stay at the level of understanding basic skills, and it is difficult to dig deep into advanced applications of tools, failing to achieve proficiency. Over time, the corresponding skills and method skills are slowly indifferent, making it difficult to give play to the design height of comprehensive applications of animation tool skills.

\section{Practice mechanism is inadequate}

The mechanism of teaching practice in colleges and universities is not complete enough, including the teaching curriculum system, assessment standards, practice-related teaching evaluation and reward and punishment mechanisms, etc. These mechanisms have an adverse impact on the teaching team, and indirectly affect teaching. Colleges and universities generally adopt a teaching system that combines research and teaching. In this way, in addition to completing basic teaching tasks, teachers must face arduous and difficult research tasks. At the same time, the reform of the teaching system and mechanism also occupies a large proportion of teachers' time and energy, coupled with the pressure of their own academic theories to be promoted and promoted, resulting in a great reduction in the quality of teaching practice. On the other hand, under the current social employment environment, students face multiple pressures and have to learn more about other professional abilities. In addition, the knowledge system of this major is updated too quickly 
and the teaching curriculum is lagging. In the face of the school examination and assessment system, as long as the examination is satisfactory, graduation can be achieved, resulting in students' unwillingness to spend time in long practice operations, making practice exercises insufficient.

\section{THE ROLE OF COMPETITIONS IN THE TRAINING OF ANIMATION CREATIVE TALENTS}

\section{A. Competition effectively inspects teaching quality}

The goal of cultivating creative talents [3] in colleges and universities is to meet the actual needs of enterprises, and their effectiveness must and can only be evaluated by the actual situation of enterprises. Most of the current competitions, such as the Chinese University Student Computer Contest, Art Design Contest, and Animation Design Contest, are mostly designed in keeping with the current social and corporate needs. There are also many thematic competitions based on the actual difficulties, job requirements, and actual problems of animation industry companies. Relatively speaking, emphasis on reality, timeliness, and practicality will test the true design ability and practice level of students and teams. It is also a test of the teaching level of colleges and universities helps to find out the difficult points and pain points of the deep-level teaching reform, and to test and improve the quality of practical teaching.

\section{B. Competition promotes the deepening of teaching reform}

At present, the competitions for college students of various majors are in full swing, and the influence of animation competitions is also increasing, which has a wide impact on teachers and students in colleges and universities. With the expansion of influence, the positive influence of competitions on teaching reform is also increasing. First of all, the competition for animation students emphasizes practicality and results. Participating colleges and universities will attach great importance to the training of their practical ability. According to the relevant requirements of the competition organizing committee, they will guide and organize students to conduct targeted training for a long time, and adjust the teaching content and mechanism through the design of activities. This deepens the degree of theoretical teaching to a certain extent, strengthens the hands-on practice, accumulates relevant teaching experience, and promotes the reform of project-based teaching mechanisms.

Secondly, through the honors and awards of the contest, the professional enthusiasm of college teachers and students has been well stimulated, expanding the professional vision and developing application scenarios. The competition activity is like opening a window to learn more about the current situation and real prospects of industry practitioners. The competition can also become a platform for teaching and learning between teachers and students in different colleges and universities, and an arena for showing results.

What's more, the organizers of the competition invited industry and enterprise practitioners to participate widely to form a big financial city that gathers competition activities, academic exchanges, and skills upgrades. Different college teams conduct indepth academic exchanges and teaching mechanism exchanges. On the basis of learning and reference, it can effectively promote and improve the "schoolenterprise cooperation, work-learning combination, production-university-research cooperation" model.

Finally, in the environment of related teaching facilities, equipment, and software tools, competitions can also be conducted to test and improve, which has gradually promoted the optimization of teaching bases, training bases, and practical environments.

\section{Competition reflects teamwork spirit}

The competition is most capable of realizing teamwork in real battles. A good work is often completed by close collaboration of the team, and an excellent work is often inseparable from the team's division of labor. The task goal of the animation competition is relatively large, and it is significantly necessary for the team members to participate collectively in order to do a good job. This will test and exercise the individual's teamwork ability.

The competition process must require the team to have a good organizational structure, division of labor and cooperation mechanism, cooperation and communication channels, which can not be learned in class. In the process of completing the work, the team will set work tasks, goals, and phased steps. The team members will work in coordination and work towards the same goal. According to their respective abilities and specialties, they undertake different aspects of work. At the same time, they emphasize cooperation and communication. At the same time, in the process of coexistence, gradually cultivate the team spirit, professional mentality and interpersonal relationship processing mechanism of team members. In the process of getting together between members, they can improve their self-cultivation. Students can also feel the ability and deficiency of self from the evaluation of others, better self-awareness, improve the sense of tacit understanding and identity between students, and lay a good foundation for professional literacy for graduation into the society. This kind of high-intensity competition relationship forces students to actively strive for practice opportunities and improve their skills. These are effects that are difficult to achieve in classroom practice. 


\section{Competition is conducive to the cultivation of creative talents}

From the content of the competition and professional applications, the university student competition emphasizes innovation, creativity, and closeness to reality. It is not only a stage for skill demonstration and improvement, but also a space for the creation of works that simulates reality. The platform is very beneficial for training students' imagination, logical thinking ability and artistic creative thinking.

\section{COMPETITION-DRIVEN ANIMATION PROFESSIONAL PRACTICE TEACHING MODE OF SCHOOL-ENTERPRISE COOPERATION AND COMBINATION OF WORK AND STUDY}

\section{A. Integrating practical teaching content by competitions [4]}

Competition has a strong penetration effect on the integration of independent skills and the integration of independent teaching modules. College courses are relatively isolated, coupled with high hands-on requirements at each stage and limited time distribution, a topic's skills often cost an entire lesson. Therefore, the teaching practice course is relatively independent and lacks coherence, and the competition can just make up for this deficiency, which is a test of integration.

There are many design and computer competitions in colleges and universities every year. Most of these competitions require students' comprehensive ability, and they are increasingly emphasizing that students should have aesthetic and humanistic skills, and comprehensively apply various animation software design tools in design technology. In recent years, large-scale college student graphic design competitions have emphasized the flexible application of a variety of software, including the mainstream three major graphic drawing software represented by Photoshop and CorelDraw; Short video design competition, participants are required to be proficient in using related software, including graphics software Photoshop, video editing, film and television cropping, audio track tools, After effects, etc.; The webpage design competition will also need to use a variety of webpage format design software of different standards, requiring students to have diverse skills. At present, the organizers of the competition are more inclined to organize competition topics that integrate multiple themes, in addition to intensifying the originality, theme expression ability, speech expression ability, etc. In addition to comprehensively inspecting design capabilities and computer application capabilities, they comprehensively inspect students' comprehensive qualities and comprehensively improve their overall strength.
In addition, the scope of the competition can also integrate film and television script, computer skills, aesthetic culture industry, because the animation major itself is a widely used profession, which involves subject knowledge in the animation industry, design science, script creativity, computer design tools, aesthetics, film and television media, advertising, cultural operations and other aspects. Through multidisciplinary and inter-disciplinary competition practice, it can fully demonstrate the multi-level knowledge system required for future employment, comprehensively expose all the shortcomings reflected by students in the school because of a single subject and a monotonous level of practice, and better remind students of the range of knowledge and skills required. In the same way, it can provide a lot of interdisciplinary useful supplements to practical teaching content.

For example, organizers can look for "contest themes" in similar cultural and creative fields, use animation skills, use animation art design tools, and combine "science popularization of science", film and television entertainment, advertising, media operations, etc. to conduct special design competitions, which helps students better grasp the relevant industries of the cultural media industry, and enhances the visibility and professional breadth of the participants; from the perspective of the animation industry chain, integrating a variety of animation expressions, focusing on the combination of "professional art and animation design", film and television script creation, new animation forms such as MG animation, etc., to design the theme of the competition from the depth of the animation profession, which helps participants to better understand the industry dynamics and explore the level of animation technology design in depth; or it can design competition topics from the perspective of cross-professional cooperation. In fact, animation majors can be combined with most other professional industries. For example, animation combined with "party courses in colleges and universities" can be used to formulate "competitions for developing teaching curriculum systems", allowing students design a whole series of "comic version of college party teaching system" or "animated version of medical science video". From these perspectives, the teaching applications related to the animation industry for students can be explored in a simple way. Similarly, for animation teaching reformers, through the organization and participation of competitions, students and teachers can fully understand the thinking and thinking behaviors of students and teachers. The results of the competition design can also be incorporated into the teaching material reform, comprehensive design and overall consideration, and systematic resource planning, curriculum design, teaching system design and other program design, which greatly promote the systematic and comprehensive teaching reform. 
Obviously, the competition integrates the comprehensive application of multiple disciplines and courses, which is an improvement of practical training. It further strengthens the students' practical ability and design thinking, and strengthens the organization and coordination and innovation and creativity of the entire team within the prescribed period. Students' hobbies and understanding of careers are conducive to better progress towards professionalization. The experience of participating in the competition enables students to exercise their comprehensive qualities and the application skills of professional software tools, and learn a variety of design methods. In practice, students experience the skills required for actual works, find gaps in them, and better summarize their experience. In addition, comparing competitors and outstanding practitioners, it is easy to find career development paths and professionalization benchmarks, so as to determine the future career development direction.

\section{B. Combining competition with practical training [5]}

School-enterprise cooperative training teaching is an effective way for colleges to adopt practical teaching, and it has now formed mature social benefits. The introduction of competition mechanism is a useful exploration and innovation, fully drawing on the good model of absorbing school-enterprise cooperation. Incorporate the content of corporate practical training, re-evaluate the assessment and evaluation standards, connect with the company's cutting-edge practical design business, and introduce a variety of competition mechanisms. Participating in corporate job skills internships based on intra-school competitions, it can also allow students to experience competition and cooperation consciousness, further inspire enthusiasm for internships, effectively form a good feedback of "competition to promote training and competition to improve learning", and combine competitions and internships to make students learn from each other. Through the competition, the school and the company understand each other more fully and communicate more fully. The school can better understand what the company needs, the company can better understand the students, and it is more willing to accept outstanding practical students to participate in the creation of the company.

Taking the west coast of Fujian Strait as an example, it has huge cross-strait enterprise resources and has many local industrial clusters in the animation industry, including cultural and creative industrial parks and animation industry bases. In the organization of the competition, industrial cluster parks or large and medium-sized animation design companies can be fully introduced, and the characteristics of localized industrial clusters can be combined through the design of the "competition theme". Fujian natives have typical characteristics of Fujian culture and Strait culture, especially Hakka culture, marine shipping administration, and sea silk culture. The content of the competition can be integrated into cultural background and regional foundations, taking institutionalization, digitalization, and platform as carriers. Integrated with modern science and technology, combined with 3D technology, virtual reality technology, film and television creativity and digital media, mainly it focus on digital animation virtual reality, comics, games and other directions for topic selection, which is bound to arouse the enthusiasm of teachers and students in colleges and universities, and is very close to the forefront of the industry, having a strong era character. Enterprises are very welcome to combine new technologies and skills with the current hot application areas, and they need practitioners with high skills and high level in the frontier fields; colleges and universities are also eager to find the frontier professional direction of society and the requirements of enterprise employment through topic competitions in hot areas, and also provide useful explorations for the direction of talent training and the goal of teaching reform mechanism. During the competition, it is relatively easy for the students with good comprehensive ability and strong professional ability to stand out. This is conducive to the discovery of outstanding seedling talents, and also facilitates the selection of talents by the enterprise, thereby reducing the personnel cost of the enterprise, increasing the employment rate of the school, and forming a virtuous circle.

\section{Strengthening the construction of competition mechanism}

In order to ensure that competition activities can achieve effective expectations, universities should strengthen the construction of competition mechanisms, strengthen competition incentives and the construction of works display platforms, strengthen the guidance of teacher and student teams, and strengthen the docking and integration with teaching mechanism reform.

The first is to ensure the fairness and fairness of the process and results of the competition. College organizers must comprehensively consider all aspects to create an atmosphere of fair competition. Colleges and universities can use the local area network of the campus and call for announcements to select topics and methods of competitions, formulate normal competition mechanisms, student jury mechanisms, and scoring systems, strengthen organizational security, and guide participating students to combine practice with subjective initiative, exercise creative thinking and improve the design level.

The second is to establish an incentive mechanism based on practical effects. Students usually participate in the competition by motivating the team, and the 
competition tasks also require extra extra-curricular time to complete, improving the compactness of learning and practice, and enhancing exercise. Colleges and universities should improve the teaching evaluation system, integrate the competition into the assessment and inspection, and give material and honorary rewards by appraising the best, the three best students and scholarships. In addition, colleges and universities should build special exhibition halls to attract more outstanding students' works. At the same time, regular competition activities should be organized to guide students to pay more attention to the current industry hotspots and professional directions, building a platform for external exhibitions as well as opening the door to social industry communication, which in turn will help improve the application of teaching results.

The third is to focus on personal future career development. Colleges and universities should consider giving certain rewards in terms of credits, course scores, employment recommendations, etc., to encourage students to participate enthusiastically. In terms of credits and grades, students can be provided with a variety of choices, and credits for the corresponding types of courses can also be selected, and scores will be added. In terms of employment recommendation and school-enterprise cooperation internships, students with excellent performance in the competition can be given preferential opportunities. Enterprises are also very willing to choose those talents with strong practical skills and better comprehensive ability.

\section{CONCluSion}

In general, in the context of college curriculum reform, professional teaching upgrade, and innovation in teaching methods, the introduction of competitions and mechanisms in colleges and universities, and the integration of teaching practice training courses can greatly activate the reform of college teaching systems. On the one hand, it can effectively stimulate the enthusiasm, initiative and creativity of students, make students closer to the needs of enterprises and society, and promote the training mode of creative talents in animation to a higher level; On the other hand, the transformation and upgrading of teaching methods has been accelerated to a large extent. The teaching methods and the construction of teaching courses have been reorganized from practice, and the pace of teaching reform has been accelerated. It is conducive to the optimization of the professionalization of the teaching team, and the teaching practice is closer to the needs of the corporate society. Multi-level construction of teaching display and corporate training platform has deepened the production-study-research and schoolenterprise cooperation model, and played an important role in the innovation of the training mode of animation and animation professional practice talents and the innovation of the teaching system.

\section{References}

[1] Liang Mingliang, Zhang Huimin, National Higher Vocational Students' Skills Competition on the Promotion of Teaching Reform and Talent Training [J]. Experimental Technology and Management, 2011, (2): 161-163. (in Chinese)

[2] Zhang Xiaoju, Lu Lu, Niu Yanfei, On the Promotion of Skil Competition to Deepen the Reform of Practical Teaching [J]. Education Exploration, 2008, (2): 37-38. (in Chinese)

[3] Yang Heng, Research on the Effect of Animation Vocational Skill Competition on Teaching Reform [J]. Chinese Vocational and Technical Education, 2012, (14): 88-91. (in Chinese)

[4] Wang Juan, Liu Yan, Exploration on the Reform of Practica Teaching Methods for Animation Major [J]. Education and Vocation, 2013, (6): 161-162. (in Chinese)

[5] Wu Jian, Thoughts on Leading Vocational Teaching Reform with Vocational Skills Competition [J]. Education Exploration, 2013, (4): 75-76. (in Chinese)

[6] Qian Daqing, Reflect the Role of Vocational Skill Contest \& Reconstruct Vocational Educational System [J]. Xingtai Vocational and Technical College, 2010, (8): 47-49. (in Chinese) 